\title{
Imaging Findings in Chest Computed Tomography: Initial Experience in a Developing Country
}

\author{
Adenike T. Adeniji-Sofoluwe1, Ademola J. Adekanmi ${ }^{1}{ }^{*}$ Richard Efidi $^{2}$ \\ ${ }^{1}$ Department of Radiology, College of Medicine, University of Ibadan, Ibadan, Nigeria \\ ${ }^{2}$ Department of Radiology, University College Hospital, Ibadan, Nigeria \\ Email: *kanmiademola@gmail.com, *aadekanmi@comui.edu.ng
}

How to cite this paper: Adeniji-Sofoluwe, A.T., Adekanmi, A.J. and Efidi, R. (2017) Imaging Findings in Chest Computed Tomography: Initial Experience in a Developing Country. Open Journal of Clinical Diagnostics, 7, 113-123.

https://doi.org/10.4236/ojcd.2017.74012

Received: October 6, 2017

Accepted: December 11, 2017

Published: December 14, 2017

Copyright $\odot 2017$ by authors and Scientific Research Publishing Inc. This work is licensed under the Creative Commons Attribution International License (CC BY 4.0).

http://creativecommons.org/licenses/by/4.0/

\begin{abstract}
Background: High resolution chest computed tomography (HRCT) is an established imaging modality that accurately assesses disease processes that are non-specific on conventional chest radiography. Chest Computed Tomography (CT) has proven invaluable in the evaluation of various diseases of the chest providing precise diagnosis that have great impact on the course of management. In this study, our aim is to evaluate the spectrum of radiological findings in all patients that had chest CTs at a tertiary health Institution in a developing Country. Method: This is a 6-year retrospective study of all patients that had Chest CT from 2009 till 2015. All patient data were retrieved and their Chest CT images reviewed and radiological findings and diagnosis documented. Results: Age range of patients was $1-84$ years with a mean of $52.36 \pm 22.9$ years. Ten patients were children $(12 \%)$. There were more males $53(63.1 \%)$ than females $31(36.9 \%)$ in the study. Referral for imaging was predominantly from internal medicine $33(47.1 \%)$ and Surgery 27 (38.6\%) departments. Most of the patients, 53 (63.1\%) did not have previous chest radiographs. Consolidative changes were the most common parenchymal finding $(77.4 \%)$. Few mediastinal masses were found in the study population. CT angiography constituted only $10.7 \%$ of all chest CTs done in adults. Conclusion: Few chest CT evaluations are performed compared to CTs of other parts of the body done in the same period. Chest $\mathrm{CT}$ is generally under-utilized even in a tertiary institution. There is a need to educate chest physicians on the role and advantages of CT use for the management of their patients.
\end{abstract}

\section{Keywords}

Chest, High Resolution Computed Tomography (CT), Findings 


\section{Introduction}

Computed Tomography (CT) has revolutionalised diagnostic imaging since its conception in 1971, and has become an integral part of daily thoracic imaging practice [1]. High resolution chest computed tomography is an established imaging modality, developed over the last decade, for accurate assessment of disease processes that are non-specific on conventional chest radiography. It combines very high contrast resolution with high spatial resolution in evaluating pulmonary diseases. It has proven invaluable in the evaluation of various disease or pathological conditions of the chest with resultant precise diagnosis that have great impact on the course of management.

It is indicated in trauma patients with high risk deceleration injuries for assessing the mediastinum for aortic and spinal injuries, some of which may appear normal on chest radiographs [2] [3]. Multidetector chest CT remains the best modality in the screening and diagnosis of chest trauma, with possible findings including parenchymal lesions such as pulmonary lacerations, pleural space abnormalities such as pneumothorax, diaphragmatic rupture, and rib fracture from blunt trauma to the chest wall [4]. Chest CT is also crucial for the staging of lung cancer and provides differential diagnosis via recognition of pattern and distribution of the disease [1]. Thin-slice multi-detector-row CT is also sensitive for the detection of lung nodules; evaluation of peripheral bronchogenic carcinoma for pleural and chest wall invasion [5] [6].

Although, chest CT angiography (CTA) is commonly employed to evaluate suspected pulmonary embolism, non-pulmonary embolism findings such as incidental pulmonary nodule or adenopathy may be found [7]. Chest CT remains an important imaging modality for the diagnosis of pulmonary septic emboli, and determining the extent of disease; by demonstrating features such as multiple peripheral nodules (in $83 \%$ of cases), a feeding vessel sign (67\%), cavitation (50\%), wedge-shaped peripheral lesions abutting the pleural (50\%), air bronchograms within nodules (28\%), and extension into the pleural space (39\%) [8].

Furthermore, high resolution chest CT has been documented to be highly useful in evaluating patients with acute or chronic chest symptoms for parenchymal lung disease such as invasive pulmonary aspergillosis, a potentially fatal disease [9]. The presence of the halo sign in this condition is an early sign that has been shown to be associated with better patient prognosis [10]. Chest CT can detect features of pleural tuberculosis and differentiate active from inactive disease; by detecting pleural rind of fluid collection. It can also show complications of the disease such as broncho-pleural fistula and chest wall involvement [11]. Bronchial tree diseases including inflammatory disorders such as bronchiectasis, obstructing lesions such as tumours, bronchial fistula as well as congenital abnormalities of the lungs, are well depicted on high resolution chest CT [12].

There is paucity of literature on local studies in this environment on imaging findings at chest computed tomography, hence emphasizing the relevance of this research. This study aims to describe the source of referral for chest CT and 
spectrum of radiological findings in UCH Ibadan, Nigeria.

\section{Materials and Methods}

\subsection{Study Design and Setting}

This is a retrospective and descriptive study, carried out at the radiology department of the University College Hospital, Ibadan, Nigeria spanning a 6-year period from 2009 to 2015.

University College Hospital (UCH) is the foremost Tertiary institution and Teaching Hospital in Nigeria, it is located in Ibadan, South Western and receives referral from South-Western states of Nigeria and beyond. The Hospital is an 850 bed hospital with occupancy rate of 55 - 60 with 56 service and clinical departments and runs 96 consultative out-patient clinics per week in 50 specialty and sub-specialty disciplines. About 150,000 new patients are seen in the various out-patient clinics every year.

\subsection{Method}

Inclusion criteria for this study were all subjects referred for Chest CT for various chest conditions, irrespective of their age and gender during the study period. Subjects that have most demographic and clinical information details recorded. Subject that had clear images of diagnostic value, devoid of artefacts with both pre and post contrast Computed Tomographic image correlates were evaluated. Those excluded were patients with scanty demographic and clinical information. Non diagnostic images were also excluded.

\subsection{Imaging}

A 64-slice Toshiba CT machine was used to acquire the images of 84 patients who had chest CT scans, irrespective of their age and gender during the study period. CT scanning of the chest was conducted with patient lying supine on the CT gantry and the scanogram acquired. Pre-contrast axial images were then acquired from the thoracic inlet to the lowest hemi-diaphragm using a breathholding technique. Post-contrast axial images were subsequently acquired using an automatic injection pump that delivered low osmolar contrast agent (Iopamidol $^{\mathrm{mm}}$ ) at a rate of between $3-5 \mathrm{ml}$ per second with $3 \mathrm{~mm}$ collimation and a pitch of $1.8 \mathrm{~mm}$ used. Reformatted sagittal and coronal images were then generated for analysis and reporting. The radiological data on Chest CT performed during the period were retrieved and independently reviewed by two certified and experienced radiologists. The degree of agreement between findings reported by each radiologist was evaluated with first ten patients and $\mathrm{K}=0.9$.

Data collection sheet was used to record extracted patients' information including their biodata, referral source, presenting complaints and clinical diagnosis. The imaging findings of the reviewed chest CT images were also recorded into the prepared data sheet. Missing data were also noted.

The collected data was entered into the statistical package for social sciences 
(SPSS) software version 20 spread sheet and analyzed. The data is presented using frequency tables, percentages and bar charts as appropriate. Chi square tests were used for statistical analysis of categorical variables. Ethical approval was not sought as secondary data was employed. However, there is HIPPA compliance with confidentiality preserved by assigning numbers to each eligible patient in place of real names.

\section{Results}

Eighty-four patients had chest CT study done from 2009-2014 at the UCH Ibadan. The age of patients ranged from $1-84$ years with a mean of $52.36 \pm 22.9$ years. Ten patients were children (11.9\%), 73 were adults $(86.9 \%)$ while a patient had no age documentation. There were 53 (63.1\%) males and 31 (36.9\%) females in the study. The majority, 33 (47.1\%) of the patients were refereed from the department of internal medicine and 27 (38.6\%) were from department of surgery. Referrals were also from radiotherapy with 2 (2.9\%) patients, 4 (5.7\%) from pediatrics; otorhinolaryngology and Obstetrics and Gynecology referred a case each. Most of the patients, 53 (63.1\%) patients did not have previous chest radiograph while $31(36.9 \%)$ had taken a chest radiograph prior to the chest CT. Abnormal chest X-ray findings were found in 29 (93.5\%) of these patients.

\subsection{Lungs}

In all patients, consolidative changes were the most common parenchymal finding seen in $77.4 \%$ of the studied patients, especially in the left lower lobe in $27.4 \%$. Overall, consolidation, lung collapse, intra-pulmonary mass and bronchiolar dilatation were predominantly seen in the left lower lobe (51.2\%). Intra-pulmonary masses alone were frequently seen in the left upper lobe (14.3\%) and the lower lobes (10.7\%) as shown in Table 1.

\subsection{Pleural}

There were more pleural collections on the left as seen in 21 (25\%) patients than on the right side 11 (13.1\%). Pneumothorax was only noted on the right side in only $2(2.4 \%)$ patients. A diagnosis of pleural based mass was made in $3.6 \%$ of patients.

\subsection{Mediastinum}

Few mediastinal masses were found in the study population. Anterior mediastinal masses were found in $3(3.6 \%)$ of the patients, posterior mediastinal mass was documented in $2(2.4 \%)$, while superior, inferior and middle mediastinum masses were seen in one patient $(1.2 \%)$ each. Soft tissue swelling was present in only $5(6.0 \%)$ patients.

\subsection{Vascular}

CT angiography constituted only $10.8 \%$ of all chest CTs done. Only $9 / 83$ adults 
Table 1. Distribution of lung lesions on CT chest.

\begin{tabular}{cccccccc}
\hline \multirow{2}{*}{ Lung findings } & \multicolumn{6}{c}{ Lung Lobes } & \multirow{2}{*}{ Total } \\
\cline { 2 - 7 } & RUL & LUL & RML & LL & RLL & LLL & \\
\hline Consolidation & $5(6 \%)$ & $9(10.7 \%)$ & $7(8.3 \%)$ & $8(9.5 \%)$ & $13(15.5 \%)$ & $23(27.4 \%)$ & $65(77.4 \%)$ \\
Collapse & $1(1.2 \%)$ & $7(8.3 \%)$ & 0 & $4(4.8 \%)$ & $3(3.6 \%)$ & $9(10.7 \%)$ & $24(28.6 \%)$ \\
Mass & $8(9.5 \%)$ & $12(14.3 \%)$ & $5(6.0 \%)$ & $8(9.5 \%)$ & $8(9.5 \%)$ & $9(10.7 \%)$ & $50(59.5 \%)$ \\
$\begin{array}{c}\text { Bronchiolar } \\
\text { Dilatation }\end{array}$ & $2(2.4 \%)$ & $4(4.8 \%)$ & $2(2.4 \%)$ & $2(2.4 \%)$ & $2(2.4 \%)$ & $2(2.4 \%)$ & $14(16.8 \%)$ \\
\hline
\end{tabular}

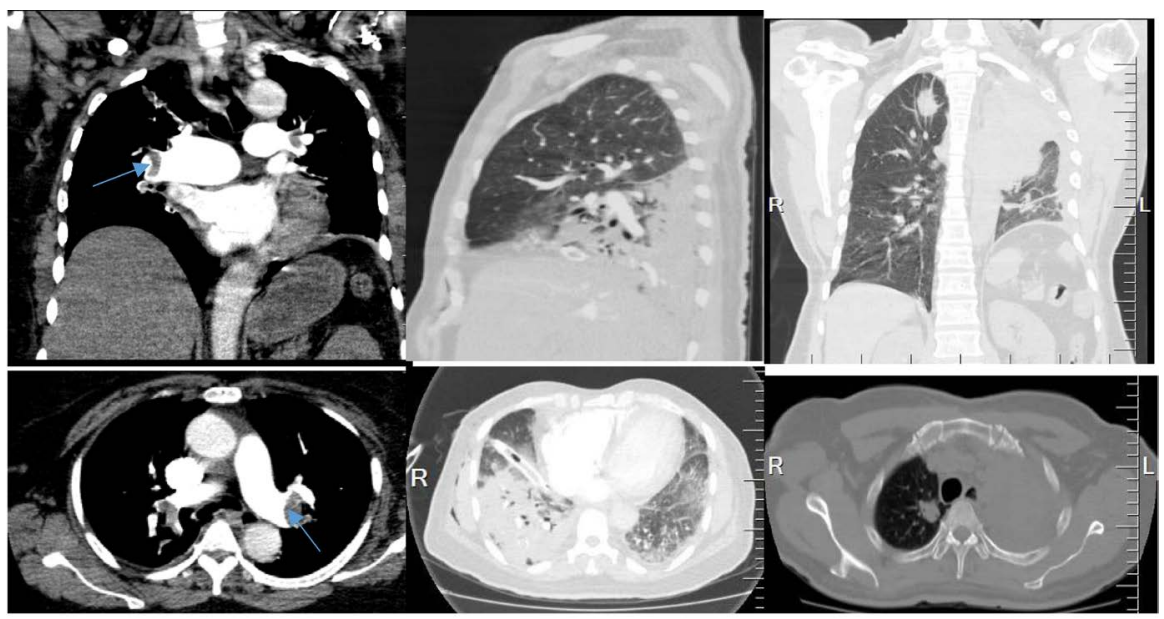

(a)

(b)

(c)

Figure 1. Spectrum of findings at Chest CT. (a) Pulmonary embolism: CT pulmonary angiography in mediastinal window, in coronal and axial views showing concave filling defects (blue arrows) in the right and left main pulmonary arteries surrounded by a rim of contrast. There is associated dilatation of both pulmonary arteries worse on the right side. Poor inspiratory effort is noted as a result of the breathlessness, (b) Consolidation: CT chest in lung window, in sagittal and axial views demonstrate extensive hyperdensity in the right lower lobe limited superiorly by the oblique fissure with branching tubular hypodensities within it (air bronchogram). A chest tube is noted in situ, (c) Bronchogenic CA: CT Chest in lung and mediastinum window in Coronal and axial views.

were referred for CTA. Aneurysmal dilatation of the ascending aorta was seen in one patient. Calcifications were noted in the ascending aorta of $5(6.0 \%)$ patients, aortic arch of 8 (9.5\%) patients and descending aorta of $6(7.1 \%)$ patients. Clots/thrombus was found in the pulmonary trunk in only one patient, in the right main trunk and left main trunk in $2(2.4 \%)$ patients (Figure 1 ).

\subsection{Vertebrae}

Only 9 reports adequately documented degenerative changes in the spine. The changes found include but are not limited to osteophytes, bone sclerosis and destruction in reducing frequency. The lower thoracic vertebrae (T6-T12) were more commonly affected than the upper thoracic vertebrae, but to varying degrees.

\subsection{Radiologic Diagnoses}

Overall, chest CT was normal in $9(11.4 \%)$ patients. The top radiologic diagnoses 


\section{Top Radiological Diagnoses}

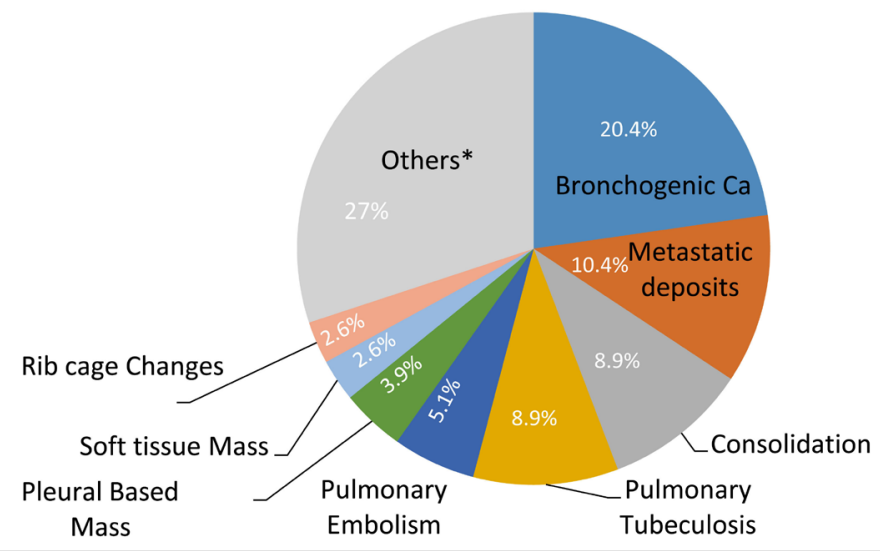

Figure 2. Top radiologic diagnoses made at chest CT of 84 patients. Others ${ }^{\star}$ : atelectasis, emphysematous bullae, interstitial lung disease, pleural effusion, pneumothorax, aspergilloma, sarcoidosis, etc.

made were bronchogenic carcinoma (20.4\%), metastatic deposits (10.4\%), consolidation (8.9\%), pulmonary tuberculosis (8.9\%), pulmonary embolism (5.1\%), pleural based mass (3.9\%), soft tissue mass (2.6\%) and rib cage changes in (2.6\%) as depicted in Figure 2. Other diagnoses (27\%) made comprised of atelectasis, ephysematuos bulla, interstitial lung disease, pneumothorax, aspergilloma and sarcoidosis, etc. All the diagnoses made were more common in males than in females. None of these diagnoses was made in children except for consolidative changes.

\section{Discussion}

CT has emerged as a useful tool in the study of the anatomy, physiology, pathology of the body [13]. CT gives good spatial resolution of both soft tissue and bony structures [14]. CT is more informative than plain radiography and helps to narrow down differential diagnoses. High resolution CT of the chest is a powerful tool for the evaluation of acute and chronic respiratory diseases [9]. For example, lung consolidation appears as foci of decreased attenuation which becomes more conspicuous on contrast-enhanced CT [15].

Low dose chest CT is regarded as the investigation of choice for the detection of lung disorders in children with persistent or progressive chest symptoms despite institution of conventional treatment [16]. In this study children accounted for only $12 \%$ of the study population indicating that few referrals were made for chest imaging. A study carried out in a children's hospital in the US evaluated 56 children who had chest CT following plain chest radiographs for persistent or progressive chest symptoms despite antibiotics therapy, found parenchymal and pleural lesions, pericardial effusion that were not apparent on plain chest radiographs [17]. Parenchymal complications like abscesses and bronchopleural fistulas 
were detected in about $70 \%$ of the cases. Pleural complications like abnormally located chest tube and loculated pleural fluid; detected easily by Chest CT were not detected by plain radiographs [17]. The volume of fluid collection was also correctly estimated by CT. Chest CTs are seldom requested to evaluate progressive or lack of response to treatment of chest diseases or complications that may arise in children in our setting. Rather requests for repeated follow-up chest radiographs are the norm. This practice might be due to several reasons like unavailability of CT facilities in the locality, lack of funds to pay for the study, inadequate knowledge of the value of chest $\mathrm{CT}$ in the management of cases of chest infection in children when $\mathrm{CT}$ is rightly indicated.

TB is endemic in Nigeria and Nigeria ranks $10^{\text {th }}$ among the 22 high-burden TB countries in the world with an estimated 320,000 prevalent cases of TB in 2010 , equivalent to $199 / 100,000$ cases [18]. The TB burden is compounded by a high prevalence of HIV and Multi-drug resistant tuberculosis (MDR-TB). Lagos, Kano, and Oyo have the highest TB prevalence rate in Nigeria. [18] [19]. In a study that evaluated 20 cases of pleural TB by Chest CT, CT was able to accurately differentiate acute from chronic disease. The diagnosis of acute disease was made with improved accuracy in areas with small cavitations and lymph node enlargement not detected by plain X-ray [11]. We are often able to differentiate active from chronic TB on plain film but not as accurately as can be done if CT is utilized for this assessment [20]. Despite these staggering epidemiologic data, and in some cases unequivocal clinical, laboratory and plain film results, screening of TB cases is often limited to plain radiographic evaluation and therapeutic trial of anti-TB drugs in our environment. The low rate of CT evaluation may be due to the managing physicians' fear of ionizing radiation and lack of adequate information of CT usage in appropriate TB cases.

High resolution chest $\mathrm{CT}$ is the most sensitive non-invasive imaging modality for evaluating parenchymal and bronchoscopically inaccessible bronchial lung abnormalities. It is indicated for symptomatic patients with normal chest radiographs and/or normal pulmonary function testing. Characterization of non-specific radiographic findings, parenchymal disease activity and follow- up, lung biopsy localization as well as to localize bronchiectasis are other documented use of Chest CT [21]. Bronchiolar dilatation was detected in 14 (16.8\%) patients in our study. Most patients with Chronic Obstructive pulmonary disease COPD are rarely referred for imaging with CT and the management of the majority of patients with COPD in our environment is often limited to clinical and plain radiographic assessment which might result in wrong classification and poor characterization of cases. Cystic fibrosis was adequately evaluated using chest CT in a study of 117 cases of cystic fibrosis conducted by Helbich et al. [22]. The study found imaging features including bronchiectasis $(80.3 \%)$, peribronchial wall thickening (76.1\%), mosaic perfusion (63.9\%), and mucous plugging $(51.3 \%)$. The value of Chest CT is further emphasized in the study by Muller et al. [23]. The study was able to determine the accuracy of chest CT in the 
recognition of bronchiectasis in 13 lungs by correlating with bronchographic findings. In this study, no case of cystic fibrosis was found, presumably due to rarity of such cases in our environment. Chest wall masses may be evaluated by plain radiography, ultrasound, CT, MRI PET/CT and guided biopsies [14]. The utilized modalities will depend on the suspected differential diagnosis. However, $\mathrm{CT}$ is regarded as the workhorse of diagnostic imaging of chest wall lesions as it gives excellent soft tissue contrast and adequately differentiates osseous from non-osseous masses [14]. Soft tissue chest wall masses such as hematomas, lipomas, liposarcomas, rhabdomyomas, elastofibromas and osseous lesions like hyperosteosis, chondrosarcomas, plasmacytomas, Schwannoma and hemangiomas have been described in the literature. In our study soft tissue masses found were few and constituted only $2.4 \%$ of all patients studied.

The United State Preventive services Task Force (USPSTF) recommends annual screening for lung cancer with low-dose computed tomography (LDCT) in adults aged 55 to 80 years who have a 30 pack-year smoking history and currently smoke or have quit within the past 15 years. There is no Lung cancer screening policy in Nigeria as is the case for most cancers. This might be due to socio-political reasons in addition to reported low incidence of cancer in local literature. In a 30-year (1965-1990) retrospective review of cases of lung cancer from the Cancer Registry of the University College Hospital (UCH), Ibadan Nigeria, only 142 cases were analyzed with a male: female ratio of 1.7:1. Squamous cell carcinoma was found most commonly but adenocarcinoma predominated in females among all lung cancers diagnosed at autopsy [24]. The epidemiology of malignant lung tumours in Ibadan at the time probably differs from that in the more industrialized countries and this suggests a different view of risk factors for this environment [24]. Also at the time CT was not an available means of investigation. Twenty years later, CT is still unavailable and inaccessible as an imaging modality and lung cancer screening tool. A recent evaluation of lung cancer in the eastern part of Nigeria seems to suggest an increasing incidence of this lesion in Nigeria with 51 new cases diagnosed over a 30-month period [25].

$\mathrm{CT}$ angiography has become a powerful diagnostic tool in neuro vascular imaging Worldwide, the newer visualization methods (2D multiplanar and the 3-D images) employed has produced images comparable to that of catheter based angiography [26]. CTA in the evaluation of pulmonary embolism has been reported to have a sensitivity range of $53-100 \%$ and specificity of $83-100 \%$ [27]. Contrast-enhanced Multidetector-row computed tomography (CT) is currently the imaging modality of choice in the evaluation of suspected pulmonary embolism or acute aortic syndrome [27] [28]. Pulmonary embolism and aortic lesions were the prominent vascular lesions detected in these study. However there is high possibility of underutilization of CTA in our environment particularly in suspected pulmonary embolism as reported in other parts of the World [27]. 


\section{Conclusion}

Few CT chest evaluations are performed compared to the total number of CTs of the other parts of the body done in the same period. Chest CT is generally under-utilized even in a tertiary institution in a typical developing country setting. There is a need to educate chest physicians/managing physicians on the role and advantage of $\mathrm{CT}$ for the management of patients. Employing low dose radiation technique based on patient size and newer software technology has reduced radiation dose up to $65 \%$ in chest examinations. Low dose chest CT is currently used for detection of lung cancers. We advocate the institution of low dose CT screening for lung cancers in our hospitals in the appropriate age group and in those with high risk factors to prevent untimely death among our population. Using the proper low-dose peadiatric settings or low dose adult dose for an individual with a justified, optimized computed tomography chest scan will result in more benefit than harm.

\section{Limitations}

This was a retrospective study and there were some missing patients'. Extracted socio-demographic and clinical information was limited to what was already in the cards.

\section{Acknowledgements}

We thank the following resident doctors: Drs. Mayowa Soneye and Omotayo Adelodun, and the entire staff of the Computed Tomography unit University College Hospital, Ibadan for their assistance and support.

\section{Conflict of Interest}

Authors declared they have no conflict of interest.

\section{Authors' Contributions}

Adeniji-Sofoluwe Adenike: Design and conception of study, supervision of acquisition of data, Analyze, evaluation of images and interpretation of data and drafting of the manuscript. Ademola J Adekanmi: Design and conception of study, supervision of acquisition of data, evaluation of images and revision of the manuscript. Efidi Richard: literature search, drafting of the introduction. All the authors have read and approved the final version of the manuscript

\section{References}

[1] Legmann, P. (1993) Imaging and Lung Disease: Uses and Interpretation. Tubercle and Lung Disease, 74, 147-158. https://doi.org/10.1016/0962-8479(93)90003-G

[2] Aaløkken, T.M., Kolbenstvedt, A. and Johansen, B. (1998) High-Resolution Computer Tomography of the Lungs. Tidsskr den Nor lægeforening Tidsskr Prakt Med ny række., 118, 2800-2804.

[3] Demetriades, D. (1998) Routine Helical Computed Tomographic Evaluation of the 
Mediastinum in High-Risk Blunt Trauma Patients. Archives of Surgery, 133, 1084-1088.

[4] Sangster, G.P., González-Beicos, A., Carbo, A.I., Heldmann, M.G., Ibrahim, H., Carrascosa, P., et al. (2007) Blunt Traumatic Injuries of the Lung Parenchyma, Pleura, Thoracic Wall, and Intrathoracic Airways: Multidetector Computer Tomography Imaging Findings. Emergency Radiology, 14, 297-310.

https://doi.org/10.1007/s10140-007-0651-8

[5] Fischbach, F., Knollmann, F., Griesshaber, V., Freund, T., Akkol, E. and Felix, R. (2003) Detection of Pulmonary Nodules by Multislice Computed Tomography: Improved Detection Rate with Reduced Slice Thickness. Emergency Radiology, 13, 2378-2383. https://doi.org/10.1007/s00330-003-1915-7

[6] Glazer, H.S., Duncan-Meyer, J., Aronberg, D.J., Moran, J.F., Levitt, R.G. and Sagel, S.S. (1985) Pleural and Chest Wall Invasion in Bronchogenic Carcinoma: CT Evaluation. Radiology, 157, 191-194. https://doi.org/10.1148/radiology.157.1.4034965

[7] Hall, W.B., Truitt, S.G., Scheunemann, L.P., Shah, S.A., Rivera, M.P., Parker, L.A., et al. (2009) The Prevalence of Clinically Relevant Incidental Findings on Chest Computed Tomographic Angiograms Ordered to Diagnose Pulmonary Embolism. Archives of Internal Medicine, 169, 1961-1965.

[8] Kuhlman, J.E., Fishman, E.K. and Teigen, C. (1990) Pulmonary Septic Emboli: Diagnosis with CT. Radiology, 174, No. 1. https://doi.org/10.1148/radiology.174.1.2294550

[9] Gotway, M.B., Reddy, G.P., Webb, W.R., Elicker, B.M. and Leung, J.W.T. (2005) High-Resolution CT of the Lung: Patterns of Disease and Differential Diagnoses. Radiologic Clinics, 43, 513-542. https://doi.org/10.1016/j.rcl.2005.01.010

[10] Greene, R.E., Schlamm, H.T., Oestmann, J.-W., Stark, P., Durand, C., Lortholary, O., et al. (2007) Imaging Findings in Acute Invasive Pulmonary Aspergillosis: Clinical Significance of the Halo Sign. Clinical Infectious Diseases, 44, 373-379. https://doi.org/10.1086/509917

[11] Hulnick, D.H., Naidich, D.P. and McCauley, D.I. (1983) Pleural Tuberculosis Evaluated by Computed Tomography. Radiology, 149, No. 3. https://doi.org/10.1148/radiology.149.3.6647852

[12] Grenier, P., Cordeau, M.P. and Beigelman, C. (1993) High-Resolution Computed Tomography of the Airways. Journal of Thoracic Imaging, 8, 213-229. https://doi.org/10.1097/00005382-199322000-00006

[13] Alfidi, R.J., Haaga, J., Meaney, T.F., MacIntyre, W.J., Gonzalez, L., Tarar, R., et al. (1975) Computed Tomography of the Thorax and Abdomen: A Preliminary Report. Radiology, 117, 257-264. https://doi.org/10.1148/117.2.257

[14] Mullan, C.P., Madan, R., Trotman-Dickenson, B., Qian, X., Jacobson, F.L. and Hunsaker, A. (2011) Radiology of Chest Wall Masses. American Journal of Roentgenology, 197, 460-470. https://doi.org/10.2214/AJR.10.7259

[15] Kanne, J.P., Yandow, D.R., Mohammed, T.L.H. and Meyer, C.A. (2011) CT Findings of Pulmonary Nocardiosis. American Journal of Roentgenology, 197, 266-272. https://doi.org/10.2214/AJR.10.6208

[16] Incidence, A., Lucaya, J., Piqueras, J., García-Peña, P., García-Macías, M. and Sotil, J. (2000) Low-Dose High-Resolution CT of the Chest in Children and Young Adults. American Journal of Roentgenology, 175, 985-992.

[17] Donnelly, L.F. and Klosterman, L.A. (1998) The Yield of CT of Children Who Have Complicated Pneumonia and Noncontributory Chest Radiography. American Journal of Roentgenology, 170, 1627-1631. https://doi.org/10.2214/ajr.170.6.9609186 
[18] Nigerian Tuberculosis Fact Sheet, 2015.

https://photos.state.gov/.../nigeria/.../January\%20Tuberculosis\%20Fact\%20Sheet.pdf

[19] Journal, P.O. (2009) Success of the Control of Tuberculosis in Nigeria: A Review. International Journal of Health Research, 2, 19-32.

[20] Wang, Y., Lin, A., Lai, Y., Chao, T., Liu, J. and Ko, S. (2003) The High Value of High-Resolution Computed Tomography in Predicting the Activity of Pulmonary Tuberculosis. I International Journal of Tuberculosis and Lung Disease, 7, 563-568.

[21] Hauser, M., Russi, E.W. and Marincek, B. (1996) High-Resolution Computerized Tomography of the Lungs: Bases, Findings, Indications. Schweiz Med Wochenschr, 126, 398-408.

[22] Helbich, T.H., Heinz-Peer, G., Eichler, I., Wunderbaldinger, P., Götz, M., Wojnarowski, C., et al. (1999) Cystic Fibrosis: CT Assessment of Lung Involvement in Children and Adults. Radiology. Radiological Society of North America, Oak Brook.

[23] Müller, N.L., Bergin, C.J., Ostrow, D.N. and Nichols, D.M. (1984) Role of Computed Tomography in the Recognition of Bronchiectasis. American Journal of Roentgenology, 143, 971-976. https://doi.org/10.2214/ajr.143.5.971

[24] Ogunbiyi, J.O. (1995) Lung Cancer at the University College Hospital, Ibadan, Nigeria. West African Journal of Medicine, 14, 50-55.

[25] Ezemba, N. and Ekpe, E.E. (2012) Challenges of Lung Cancer Management in a Developing Country. Nigerian Journal of Medicine, 21, 214-217.

[26] Lell, M.M., Anders, K., Uder, M., Klotz, E., Ditt, H., Vega-Higuera, F., Boskamp, T., Bautz, W.A. and Tomandl, B.F. (2006) Newer Techniques in CT Angiography. RadioGraphics, 26, S45-S62.

[27] Wittram, C., Maher, M.M., Yoo, A.J., Kalra, M.K., Shepard, J.-A.O. and McLoud, T.C. (2004) CT Angiography of Pulmonary Embolism: Diagnostic Criteria and Causes of Misdiagnosis. RadioGraphics, 24, 1219-1238 https://doi.org/10.1148/rg.245045008

[28] Butler, K.H. and Swencki, S.A. (2006) Chest Pain Clinical Assessment. Radiologic Clinics, 44, 165-179. https://doi.org/10.1016/j.rcl.2005.11.002 